\title{
DEALING WITH MULTIPLE CONTACTS IN A HUMAN-IN-THE-LOOP APPLICATION
}

\author{
Daniel Dopico $^{\star}$, Alberto Luaces ${ }^{\star}$, Manuel Gonzalez ${ }^{\star}$ and Javier Cuadrado ${ }^{\star}$ \\ ${ }^{\star}$ Escuela Politecnica Superior \\ Universidad de A Coruña, Mendizabal s/n, 15403 Ferrol, Spain \\ e-mails: ddopico@udc.es, aluaces@udc.es, 10lo@cdf.udc.es, \\ javicuad@cdf.udc.es \\ web page: http://lim.ii.udc.es/index.en.html
}

Keywords: Multibody dynamics, real-time, contact.

\begin{abstract}
This paper deals with continuous contact force models applied to the human-in-theloop simulation of multibody systems, but the results are valid in general to all that applications with contacts when real-time is required. The contact model proposed in this work, is suited to collisions between massive solids for which the assumption of quasi static contact holds and it can be supposed that the deformation is limited to a small region of the colliding bodies while the remainder of them are assumed to be rigid. The model consist of two components: normal compliance with nonlinear viscoelastic model based on Hertz law, and tangential friction force based on Coulomb's law including sticktion. Furthermore, the model takes into account the geometry and the material of the colliding bodies. The tangential model presents original contributions while the normal model is completely taken from previous works. For this work, the formulation of the equations of motion is an augmented Lagrangian with projections of velocities and accelerations onto their constraints manifolds and implicit integrator. The whole solution proposed is tested in two applications: the first one is the simulation of a spring-mass system with Coulomb's friction, which is an academic problem with known analytical solution; the second one is a simulator of a hydraulic excavator Liebherr A924, which is a realistic application that gives an idea of the capabilities of the method proposed.
\end{abstract}




\section{INTRODUCTION}

The treatment of contact forces is a key issue in many applications involving multibody systems with eventual impacts or permanent contacts between bodies. If the application to address includes, moreover, human-in-the-loop, this treatment has to be even more careful because the real-time requirements impose firm constraints on the integration time step and, additionally, on the number of iterations if implicit integration is used. In addition to the efficiency considerations, the simulation has to be stable and robust enough along all the range of possible operations of the system, as well as reproduce the behavior of the real system with an acceptable precision.

The methods to solve the impact problem in multibody systems can be divided in two families (Ref. [1] Ref. [2], Ref. [3]): the discontinuous and the continuous approaches. The discontinuous approaches assume that the impact occurs instantaneously and changes the momenta balances of the system instantaneously, for this reason they are not valid to deal with contacts of finite duration or permanent contacts. On the other hand the continuous approaches are based on regularized force models that relate the force and deformation of the bodies in collision, Ref. [4], or based on unilateral constraints techniques that avoid the penetration between bodies, Ref. [5]. In applications in which it is expected to occur permanent contacts or at leasts contacts of a significant duration, continuous methods are needed and there are a number of viscoelastic and viscoplastic models that fit this category (Ref. [6], Ref. [7] ).

Between the large number of formulations of the equations of motion existent (see e.g. Ref. [8]), the penalty and augmented Lagrangian formulations Ref. [9], Ref. [10] are characterized by transforming the constraints into forces proportional to the constraints violation. This technique, used along this work, is similar and compatible to that of the continuous force models for normal contact, which relate the force and deformation of the bodies in contact to avoid the penetration between them.

It is worth to mention that, up to these days, there is not a universally accepted model to calculate the friction force between bodies under dry conditions. The Coulomb's friction law is the most simple model but has the problem that the gradient of the force at null tangential velocity is infinite. This fact is unacceptable from the numerical point of view, since the motion has to be solved in discrete time steps and it is not possible to deal with an infinite gradient at null velocity in these conditions (see e.g. Ref. [11]). The solution is to avoid the discontinuity of the Coulomb's model but maintaining that physical characteristics of the friction phenomenon important for the application to deal with (Ref. [16], Ref. [3]). For this paper a tangential friction model was developed to deal with the applications tackled, the model includes Coulomb's friction and sticktion at low velocities. Including the sticktion is indispensable for applications like the excavator simulator presented here, since the excavator has to work on its legs and blade, for example, on slopes.

Related to the contact models, there are two difficult problems to address in real-time applications, especially when using constant integration time step, which is the case here: the first one is the fact that the contact takes place in a limited, and sometimes very reduced, number of time steps, so that the algorithm has to be robust enough to overcome hard impacts; the second one was mentioned before and is related to the stability of the friction forces at low velocities and the transition between sliping and sticking.

This work explains the treatment chosen by the authors to address the contact between bodies in an excavator simulator for training personnel, which is an application that suffer from all the restrictions mentioned in the last paragraphs. The solution fulfills all the requirements proposed 
in terms of efficiency, robustness, and precision in the physical behavior.

\section{DESCRIPTION OF THE MULTIBODY FORMALISM}

The multibody formulation chosen for this work, is an index-3 augmented Lagrangian with projections of velocities and accelerations onto the constraints manifolds. The modelization of the systems is done in natural coordinates Ref. [8]. The formalism was extensively described in Ref. [12], for this reason only a brief summary is given here.

The equations of motion are given by the following expressions,

$$
\begin{gathered}
\mathbf{M} \ddot{\mathbf{q}}+\boldsymbol{\Phi}_{\mathbf{q}}^{\mathrm{T}} \boldsymbol{\lambda}^{*}+\boldsymbol{\Phi}_{\mathbf{q}}^{\mathrm{T}} \alpha \boldsymbol{\Phi}=\mathbf{Q}(\mathbf{q}, \dot{\mathbf{q}}) \\
\boldsymbol{\lambda}_{i+1}^{*}=\boldsymbol{\lambda}_{i}^{*}+\alpha \boldsymbol{\Phi}_{i+1}, i=0,1,2, \ldots
\end{gathered}
$$

where $\mathrm{M}$ and $\mathrm{Q}$ are the mass matrix and generalized forces vector, $\mathbf{q}$ is the vector of natural coordinates of the system, $\boldsymbol{\Phi}$ and $\boldsymbol{\Phi}_{\mathrm{q}}$ are the constraints vector and its Jacobian matrix respectively and $\boldsymbol{\lambda}$ is the Lagrange multipliers vector. The scalar $\alpha$ is the penalty factor while the index $i$ stands for the iteration number.

To integrate the equations of motion, Eq. (1), the single-step trapezoidal rule is going to be used. Since the mentioned integrator is implicit, establishing the dynamic equilibrium at time step $t+h$ (being $t$ the simulation time and $h$ the time step) leads to a nonlinear system of equations in the coordinates $q$ for the time step. In order to obtain the solution of this nonlinear system, the Newton Raphson method may be applied,

$$
\begin{gathered}
{\left[\frac{\partial \mathbf{f}(\mathbf{q})}{\partial \mathbf{q}}\right]_{t+h}^{i} \Delta \mathbf{q}_{t+h}^{i+1}=-[\mathbf{f}(\mathbf{q})]_{t+h}^{i}} \\
\mathbf{q}_{t+h}^{i+1}=\mathbf{q}_{t+h}^{i}+\Delta \mathbf{q}_{t+h}^{i+1}
\end{gathered}
$$

where $\mathbf{f}(\mathbf{q})$ and $\left[\frac{\partial \mathbf{f}(\mathbf{q})}{\partial \mathbf{q}}\right]$ stand for the residual and tangent matrix of the nonlinear problem and have the following expressions.

$$
\begin{aligned}
& \mathbf{f}(\mathbf{q})=\frac{h^{2}}{4}\left(\mathbf{M} \ddot{\mathbf{q}}+\boldsymbol{\Phi}_{\mathbf{q}}^{\mathrm{T}} \lambda^{*}+\boldsymbol{\Phi}_{\mathbf{q}}^{\mathrm{T}} \alpha \mathbf{\Phi}-\mathbf{Q}\right) \\
& {\left[\frac{\partial \mathbf{f}(\mathbf{q})}{\partial \mathbf{q}}\right] \cong \mathbf{M}+\frac{h}{2} \mathbf{C}+\frac{h^{2}}{4}\left(\boldsymbol{\Phi}_{\mathbf{q}}^{\mathrm{T}} \alpha \boldsymbol{\Phi}_{\mathbf{q}}+\mathbf{K}\right)}
\end{aligned}
$$

In Eq. (6) the matrices $\mathbf{K}$ and $\mathbf{C}$ collect the contributions of the generalized forces vector derivatives to the tangent matrix, and can be thought as stiffness and damping matrices of the system:

$$
\begin{aligned}
& \mathbf{K}=-\frac{\partial \mathbf{Q}}{\partial \mathbf{q}} \\
& \mathbf{C}=-\frac{\partial \mathbf{Q}}{\partial \dot{\mathbf{q}}}
\end{aligned}
$$

Once the convergence is achieved by Eq. (3) and Eq. (4), the velocities and accelerations obtained from the Newton-Raphson iteration, which are called here $\dot{\mathbf{q}}_{t+h}^{*}$ and $\ddot{\mathbf{q}}_{t+h}^{*}$, are projected 
onto the constraints manifolds to obtain their cleaned counterparts, $\dot{\mathbf{q}}_{t+h}$ and $\ddot{\mathbf{q}}_{t+h}$, by means of the following expressions,

$$
\begin{gathered}
{\left[\frac{\partial \mathbf{f}(\mathbf{q})}{\partial \mathbf{q}}\right] \dot{\mathbf{q}}=\left(\mathbf{M}+\frac{h}{2} \mathbf{C}+\frac{h^{2}}{4} \mathbf{K}\right) \dot{\mathbf{q}}^{*}-\frac{h^{2}}{4} \boldsymbol{\Phi}_{\mathbf{q}}^{\mathrm{T}} \alpha \boldsymbol{\Phi}_{t}} \\
{\left[\frac{\partial \mathbf{f}(\mathbf{q})}{\partial \mathbf{q}}\right] \ddot{\mathbf{q}}=\left(\mathbf{M}+\frac{h}{2} \mathbf{C}+\frac{h^{2}}{4} \mathbf{K}\right) \ddot{\mathbf{q}}^{*}-\frac{h^{2}}{4} \boldsymbol{\Phi}_{\mathbf{q}}^{\mathrm{T}} \alpha\left(\dot{\boldsymbol{\Phi}}_{\mathbf{q}} \dot{\mathbf{q}}+\dot{\boldsymbol{\Phi}}_{t}\right)}
\end{gathered}
$$

being $\boldsymbol{\Phi}_{t}$ the partial derivatives of the constraints vector with respect to time.

\section{DESCRIPTION OF THE CONTACT MODEL}

The contact forces approach proposed for this work comprises two different models: the normal force model and the tangential force model. The two models are presented separately in subsequent sections. The tangential model presents original contributions while the normal model is completely taken from previous works.

As will be described later, in the applications tackled in this paper the multibody models studied are divided in primitive objects (in the majority of the cases spheres) for contact detection purposes, and interact with CAD environments composed of triangular meshes. Under these circumstances, all the contacts can be approximated as contacts between primitives and plane surface bodies. For simplicity the case of spheres against plane surface bodies is the only case that will be explained here.

\subsection{Normal force model}

In order to choose the normal force model, some tests were done with several continuous viscoelastic models, like the Hunt-Crossley model, Ref. [4], the Lankarani-Nikravesh model, Ref. [13], and the Kelvin-Voight model, Ref. [14]. The results shown by the Hunt-Crossley and Lankarani-Nikravesh models where similar and very satisfactory while the Kelvin-Voight model suffered from a lack of dissipation in hard impacts that must be solved in few time steps. Finally, the normal force model chosen for this work was the Hunt-Crossley model Ref. [4]. The model is suited to collisions between massive solids for which the assumption of quasi static contact holds and it can be supposed that the deformation is limited to a small region of the colliding bodies while the remainder of them are assumed to be rigid. The expression for the normal force after some calculations has the following form,

$$
\mathbf{F}_{\text {rad }}=k_{\text {rad }} \delta^{n}\left(1+\frac{3(1-\epsilon)}{2} \frac{\dot{\delta}}{\dot{\delta}_{0}}\right) \mathbf{n}_{f l}
$$

where $k_{\text {rad }}$ is the equivalent stiffness of the contact and depends on the shape and material properties of the colliding bodies, $\delta$ is the indentation, $\dot{\delta}_{0}$ is the relative normal velocity between the colliding bodies when the contact is detected, $\epsilon$ is the coefficient of restitution, and $\cdot \mathbf{n}_{f l}$ is the direction of the force (see Fig.(1) $)$.

The value of $k_{\text {rad }}$ can be calculated for general colliding paraboloids but, as was mentioned before, for the applications presented here all the contacts can be approximated as contacts between spheres and plane surface bodies in which case the expression for the stiffness can be expressed by (see for example [15]),

$$
k_{\text {rad }}=\frac{4}{3\left(\sigma_{s p h}+\sigma_{p l n}\right)} \sqrt{R_{s p h}}
$$



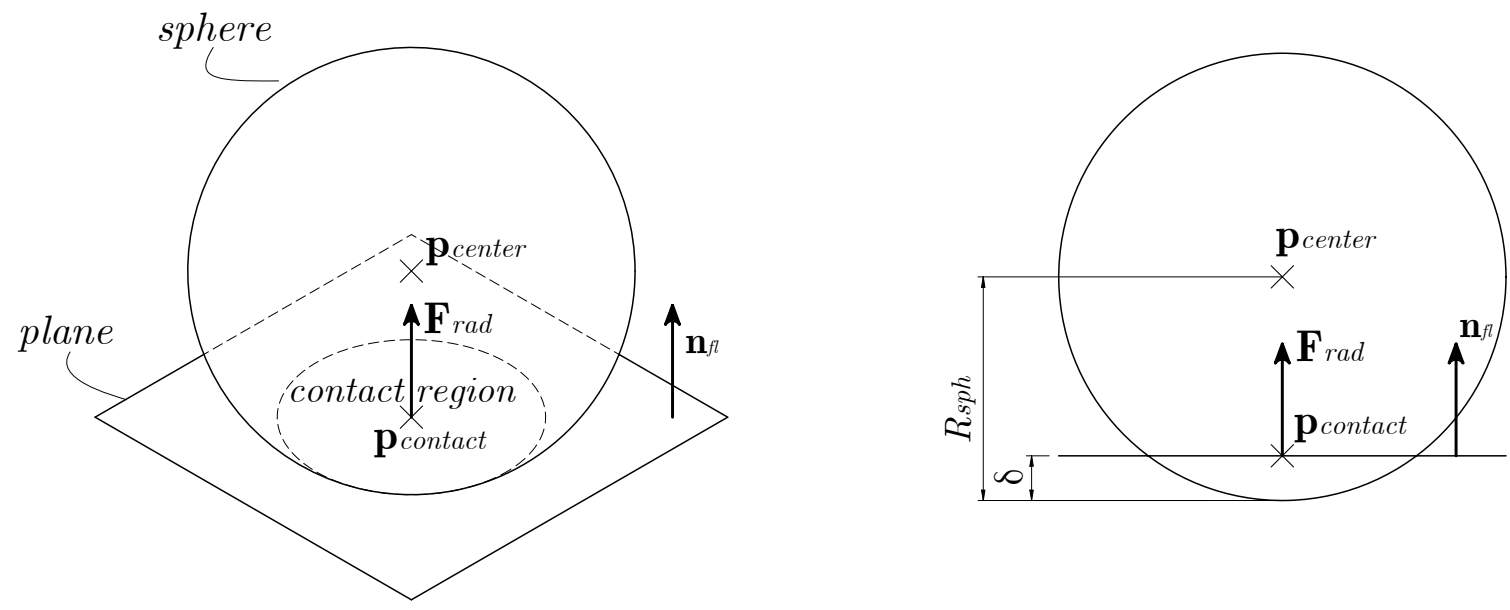

Figure 1: Normal contact between sphere and plane: isometric and front views.

where $R_{s p h}$ is the radius of the sphere in contact with the plane, and the material parameters of the sphere and plane, $\sigma_{s p h}$ and $\sigma_{p l n}$ are given by,

$$
\sigma_{s p h}=\frac{1-\nu_{s p h}^{2}}{E_{s p h}} ; \quad \sigma_{p l n}=\frac{1-\nu_{p l n}^{2}}{E_{p l n}}
$$

and $\nu$ and $E$ stand for the Poisson's ratio and the Young's modulus of each one of the two materials, represented by the sphere and plane.

\subsection{Tangential force model}

The tangential force model developed for the dry friction is based on Coulomb's law including sticktion. For the smooth transition between sticking and slipping the total force is divided in two components coupled by a smooth function, following the ideas proposed in Ref. [16]. The general form of this force is the following,

$$
\mathbf{F}_{\text {roz }}=\kappa \mathbf{F}_{\text {stick }}+(1-\kappa) \mathbf{F}_{\text {slide }}
$$

where $\mathbf{F}_{\text {stick }}$ and $\mathbf{F}_{\text {slide }}$ are the components of the sticktion and slipping forces, $\kappa$ is a smooth function of the tangential velocity, $\mathbf{v}_{t}$, which is defined in terms of the central point of the contact region, $\mathbf{p}_{\text {contact }}$, and the normal vector at the contact, $\mathbf{n}_{f l}$, as follows.

$$
\mathbf{v}_{t}=\dot{\mathbf{p}}_{\text {contact }}-\left(\mathbf{n}_{f l}^{\mathrm{T}} \cdot \dot{\mathbf{p}}_{\text {contact }}\right) \mathbf{n}_{f l}
$$

The mentioned function, $\kappa$, has to match the following conditions,

$$
\kappa=\left\{\begin{aligned}
0 ; & \left|\mathbf{v}_{t}\right|>>v_{\text {stick }} \\
1 ; & \left|\mathbf{v}_{t}\right|=0
\end{aligned}\right\}
$$

where $v_{\text {stick }}$ is a parameter of the model accounting for the velocity of the stick-slip transition. A good choice for the transition function $\kappa$ was given in Ref. [16] and has the following form.

$$
\kappa=\mathrm{e}^{-\left(\mathbf{v}_{t}^{\mathrm{T}} \mathbf{v}_{t}\right) / v_{s t i c k}^{2}}
$$




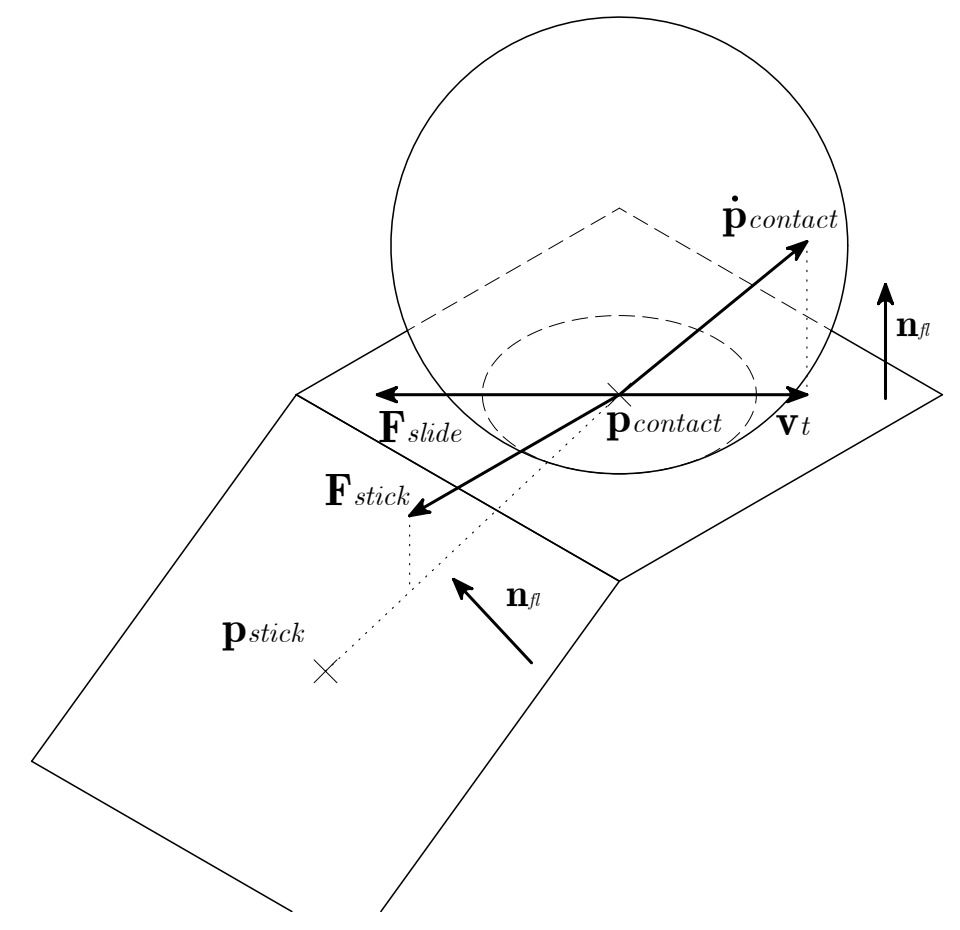

Figure 2: Tangential contact between sphere and plane.

Eq. (14) showed that the total force is composed of two contributions: the sliding friction force at high velocities and the sticktion force of the bristles at low velocities. These components have the expressions given in Eq. (18) and Eq. (19) (see Fig.(2)).

$$
\begin{gathered}
\mathbf{F}_{\text {slide }}=\left\{\begin{array}{cc}
0 ; & \left|\mathbf{v}_{t}\right|=0 \\
-\mu\left|\mathbf{F}_{\text {rad }}\right| \frac{\mathbf{v}_{t}}{\left|\mathbf{v}_{t}\right|} ; & \left|\mathbf{v}_{t}\right|>0
\end{array}\right\} \\
\mathbf{F}_{\text {stick }}=\left\{\begin{array}{cc}
0 ; & s=0 \\
\frac{f_{\text {stick }}^{m}}{s}\left(\mathbf{I}_{3}-\mathbf{n}_{f l} \mathbf{n}_{f l}^{\mathrm{T}}\right)\left(\mathbf{p}_{\text {contact }}-\mathbf{p}_{\text {stick }}\right) ; & s>0
\end{array}\right\}
\end{gathered}
$$

being $\mu$ the friction coefficient, $s=\left|\mathbf{p}_{\text {contact }}-\mathbf{p}_{\text {stick }}\right|$ the deformation of the bristles, with $\mathbf{p}_{\text {stick }}$ the central point of the bristles which was initially the central point of the contact region in the instant in which the contact began; $\mathbf{I}_{3}$ is the identity matrix of size $3 \times 3 ; f_{\text {stick }}^{m}$ is the function that represents the behavior of the bristles,

$$
f_{\text {stick }}^{m}=-k_{\text {stick }} s-c_{\text {stick }} \dot{s}
$$

being $k_{\text {stick }}$ and $c_{\text {stick }}$ the stiffness and damping coefficients of the sticktion model. Nevertheless there is a limiting value for the sticktion force.

$$
\left|\mathbf{F}_{\text {stick }}\right| \leq \mu \xi_{\text {strib }}\left|\mathbf{F}_{\text {rad }}\right|
$$

In Eq. (21) $\xi_{\text {strib }}\left(\xi_{\text {strib }} \geq 1\right)$ is a coefficient that accounts for the Stribeck effect (decreasing friction with increasing velocity). In case this limit is exceeded, and Eq. (21) is not fulfilled, there are two consequences: first, the Eq. (20) is not valid anymore and a different expression 
holds for the behavior of the bristles Eq. (22); and second, the central point of the bristles has to be updated Eq. (23).

$$
\begin{aligned}
f_{\text {stick }}^{m} & =\frac{-\mu \xi_{\text {strib }}\left|\mathbf{F}_{\text {rad }}\right| s}{\left|\left(\mathbf{I}_{3}-\mathbf{n}_{f l} \mathbf{n}_{f l}^{\mathrm{T}}\right)\left(\mathbf{p}_{\text {contact }}-\mathbf{p}_{\text {stick }}\right)\right|} \\
\mathbf{p}_{\text {stick }} & =\mathbf{p}_{\text {contact }}-\left(\frac{\eta_{\text {stick }} \mu\left|\mathbf{F}_{\text {rad }}\right|}{k_{\text {stick }}}\right) \frac{\mathbf{v}_{t}}{\left|\mathbf{v}_{t}\right|}
\end{aligned}
$$

The coefficient $\eta_{\text {stick }}$ controls the strain of the bristles when the maximum force is reached. Phisically the more reasonable value is $\eta_{\text {stick }}=1$, but small variations with $\eta_{\text {stick }}<1$ can improve the numerical behavior of the model.

\section{Contacts detection technique and computational aspects}

The methods developed in this work are designed for applications in which contact plays an important role and moreover the multibody model or models has to interact with complex CAD environments. This is the case of the simulators of certain kind of machinery and vehicles.

To deal with the kind of applications mentioned, the technique used here is to approximate the environments and the multibody models by primitive objects: the complex CAD environments by meshes of triangular faces and the geometry of the multibody systems by spheres of different sizes (in the majority of the cases) and in some cases by boxes (when the geometry cannot be approximated by spheres in a satisfactory way). Each face of the CAD environments has its own normal vector and its own properties of stiffness and friction and each sphere is characterized also depending on the material properties and curvature of the multibody model.

The first step previous to the evaluation of the forces is to detect which faces of the environment collide with geometry of the multibody model. In Fig.( 3) the different possibilities considered for contacts between primitive spheres and triangular faces are represented. The case of primitive boxes will not be explained here:

- The primitives are in contact when the distance, $s$, of the center of the sphere to the plane of the face is smaller than the radius of the sphere, $R_{s p h}$, and the projection of its center on the plane lies inside the triangle (see Fig.(3,a)). In this case there is a contact between sphere an plane and the normal vector of the contact $\mathbf{n}_{f l}$ is the normal vector of the plane of the face.

- When the previous conditions are not fulfilled, the primitives can be also in contact when the distance, $s$, of the center of the sphere to one of the edges of the face is smaller than the radius of the sphere, $R_{s p h}$, and the projection of the center on the edge lies inside the edge (see Fig.(3,b)). In this case there is a contact between sphere an edge and the normal vector of the contact $\mathbf{n}_{f l}$ has the direction from the contact point to the center of the sphere.

- If no one of the previous situations happen, it is considered that the primitives are not in contact (see Fig.(3,c) and Fig.(3,d)). It is important to notice that the collisions between sphere and vertex are neglected here. This is an acceptable approximation, but can lead to problems in environments with a lot of sharp points and non-convex geometry.

At each time step, the contact conditions have to be detected in a fast way. This involves to check all the spheres against all the faces and their edges. These are a lot of calculations per time 

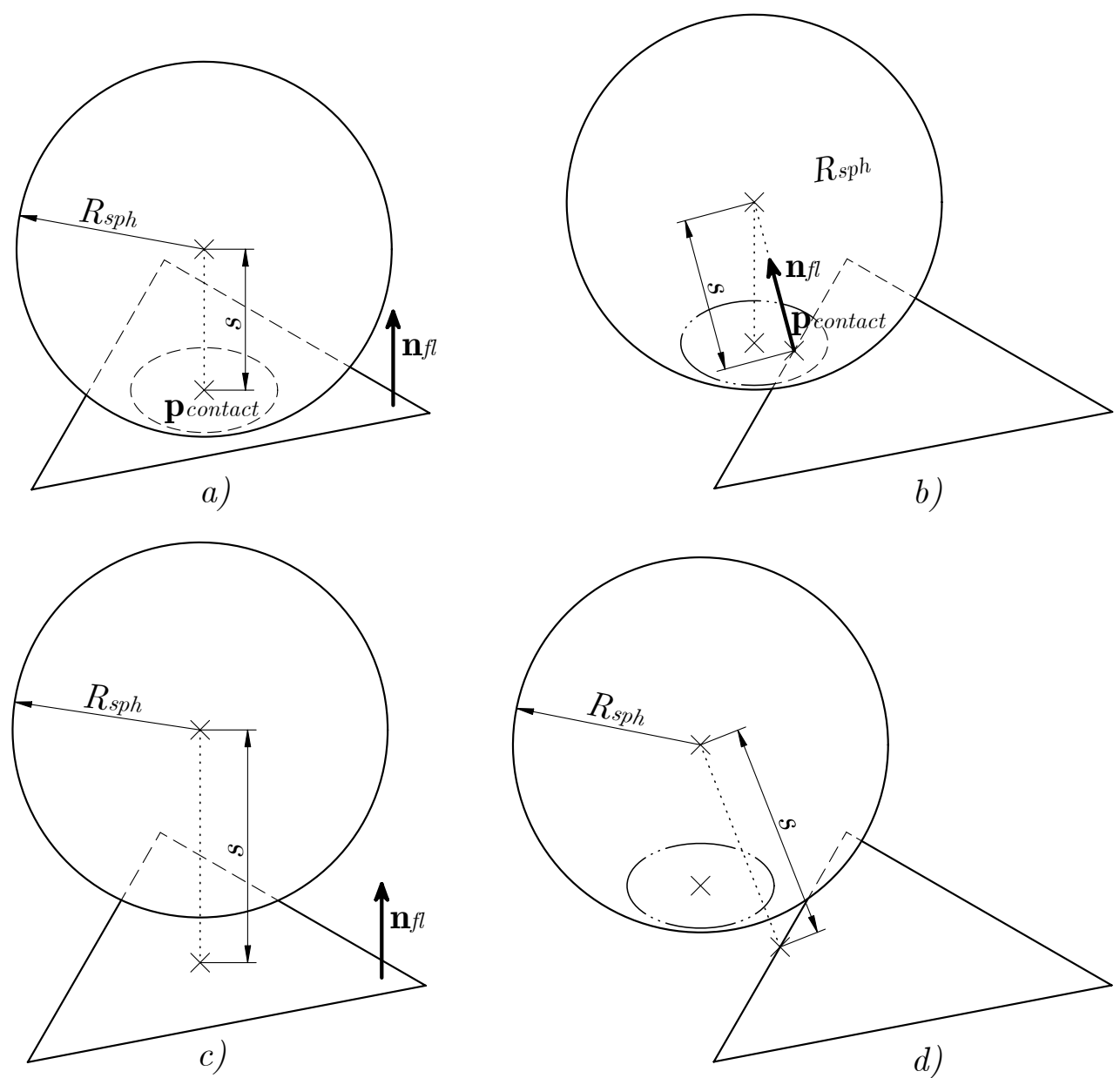

Figure 3: Colision detection between sphere and triangular face.

step when the environments are realistic. In order to speed up this process the algorithm uses an octree based hierarchical decomposition of the entire scene mesh Ref. [17]. The bounds of the polygon soup are calculated through an Axis Aligned Bounding Box (AABB) to generate a tree-based hierarchical structure that is used to quickly reject the polygons not involved in potential collisions, in order to reduce the number of polygons tested against contact with the primitive objects that represent the models geometry. The depth of the tree has to be empirically optimized for speed.

In addition, also in order to save computational time, all the calculations necessary for computing the detections are reduced to the minimum. In this sense many calculations are precomputed like: all the equations of the planes of the meshes, certain constant expressions used to decide if a point belonging to a plane is as well contained in the triangle of the face, the equations of the semi-infinite straight lines of the edges, and so on.

All the mentioned techniques are not enough for real-time purposes. As was described in section 2, an implicit integrator is used with the aim of improving the stability of the integration and consequently the algorithm is iterative, what means that if all the calculations related to the contact detection were carried out in each iteration, the cost of the detection would rise in an uncontrollable manner as the number of iterations grow. The consequences of this rise could 
be disastrous, because when the integration is more difficult the number of iterations grows and consequently both the computational cost of the dynamics and contact detection would grow as well. This is completely unacceptable. To avoid the testing of the whole tree of faces in each iteration, the faces susceptible of collision are selected after the prediction stage of the integrator and kept during the whole time step, this means once per time step instead once per iteration, what helps to maintain the computational cost per time step more constant. Depending on the number of primitives present in the multibody model, the parallelization of the contact detection must be considered also.

\section{NUMERICAL EXAMPLES}

The formulation with the contact model proposed is tested in two different applications: the first one is the simulation of a spring-mass system with Coulomb's friction, which is an academic problem with known analytical solution; the second application is the simulation of the whole model of a hydraulic excavator Liebherr A924, implemented as part of an excavator simulator, which is a realistic example that gives an idea of the capabilities of the method and the behavior of the simulations.

\subsection{Mass-spring system with Coulomb's friction}

The first system to be simulated is the mass-spring system with Coulomb's friction shown in Fig. (4), which is a very simple example with known analytical solution but at the same time interesting, to test the tangential contact model proposed, and to compare it with known theoretical results.

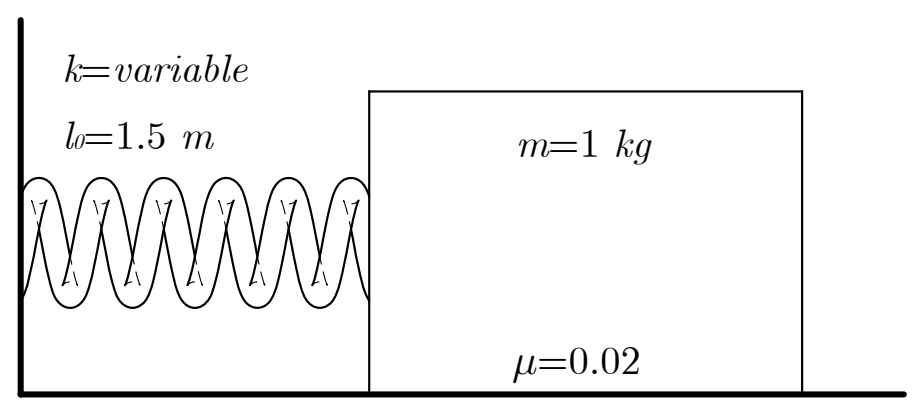

Figure 4: Mass-spring system with Coulomb's friction.

The simulation total time is 13 seconds and the time step $h=0.01$ seconds. The system undergoes the influence of the gravity forces $g=9.81 \mathrm{~m} / \mathrm{s}^{2}$. The numerical values of the parameters are: the mass $m=1 \mathrm{~kg}$, the Coulomb's friction coefficient $\mu=0.02$ the natural spring lenght $l_{0}=1.5 \mathrm{~m}$, the stiffness coefficient of the spring is $k=\left\{\begin{array}{ll}1 \mathrm{~N} / \mathrm{m} ; & t<10 \mathrm{~s} \\ 10 \mathrm{~N} / \mathrm{m} ; & t \geq 10 \mathrm{~s}\end{array}\right\}$, being $t$ the integration time. The change on the spring stiffness is motivated to force the stickslip transition when the simulation time reaches $t=10 \mathrm{~s}$, just before this instant the mass was stuck to the plane and the change in the spring stiffness forces the mass to move.

The rest of the parameters for the tangential contact model described in section 3.2 have the following values: $v_{\text {stick }}=N \mu g h, k_{\text {stick }}=m /(N h)^{2}, c_{\text {stick }}=2 \sqrt{k_{\text {stick }} m}, \eta_{\text {stick }}=1$. The parameter $N$ allows to estimate the rest of parameters of the model. Intends to be the number of 
time steps to stop the mass once the sticktion is acting, and is set to $N=5$ for this application. Excessively low values lead to numerical problems.
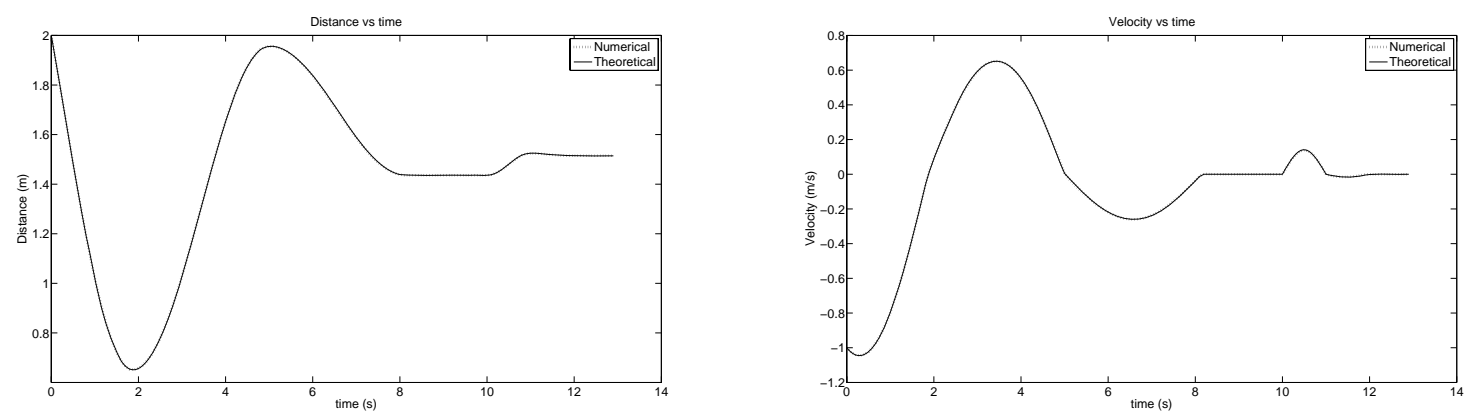

Figure 5: Response of the system (spring distance and velocity): theoretical vs. numerical.

The theoretical responses of the system vs. the numerical responses are shown in Fig.( 5), the magnitudes represented are the spring distance and velocity. The coincidence between the theoretical and numerical responses is quite good, and the slip-stick and stick-slip transitions are also satisfactory.

\subsection{Liebherr A 924 excavator}

The modeled machine is a Liebherr A924 Litronic, a medium-size wheeled excavator. It has been modeled with 14 rigid bodies and 13 revolute joints, shown in Fig.(6).Elements crucial for stability like the front stabilizer blade and the left and right lateral outriggers (rear retractable legs) have been included in the model. Hydraulic cylinders have been modeled as kinematic constraints, since the dynamics of the hydraulic circuit has not been considered in this version of the simulator.

Kinematics of the multibody system has been modeled with natural coordinates, Ref. [8]), plus some distance and angular coordinates. The resulting excavator model has 154 coordinates (including 6 distances and 7 angles) and 154 constraints (10 of them are redundant). All the technical parameters of the real machine were taken from information provided by the manufacturer, Ref. [19]). The time step chosen for the simulator is 5 miliseconds and the number of iterations of the implicit integrator is limited to 11 to guarantee the real-time performance,
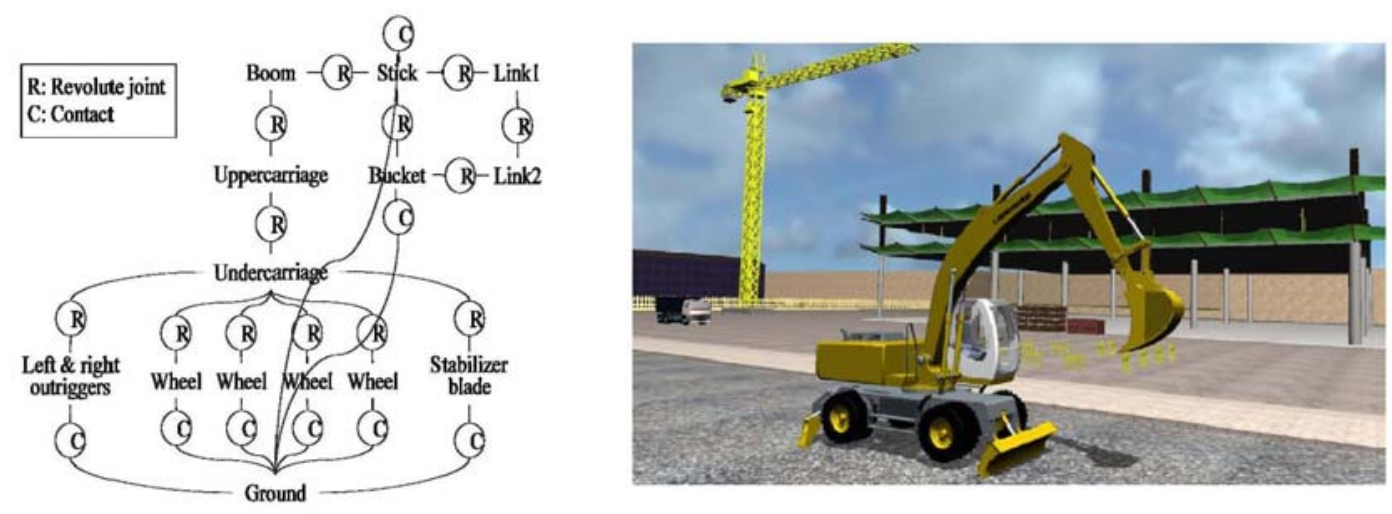

Figure 6: Topology diagram of the excavator and virtual excavator in its environment. 

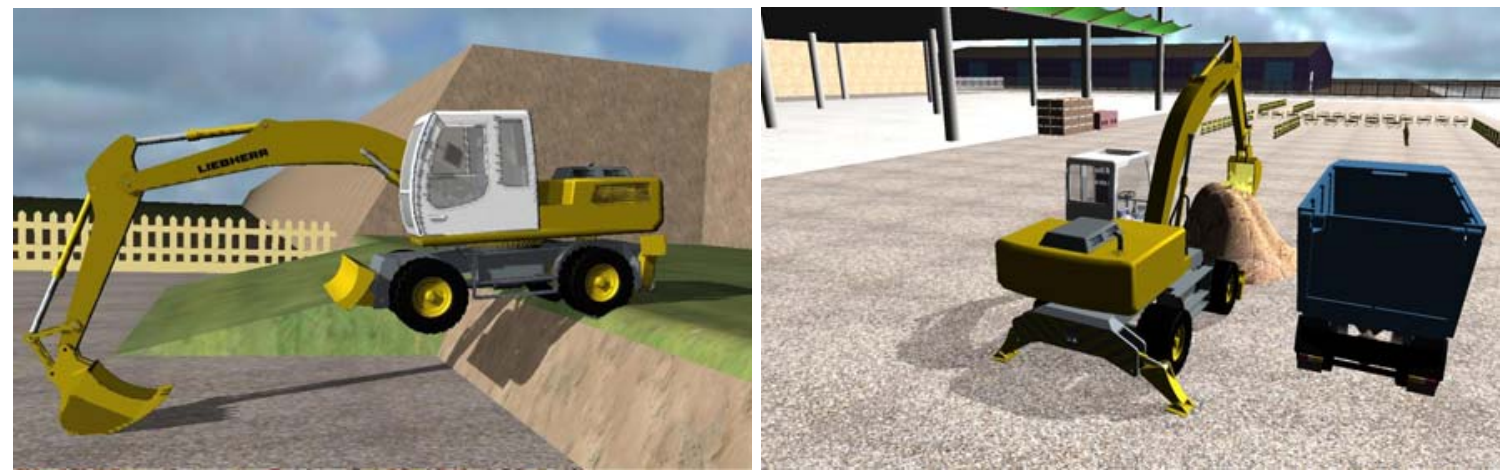

Figure 7: Using the arm for descending a steep slope and terrain excavation.
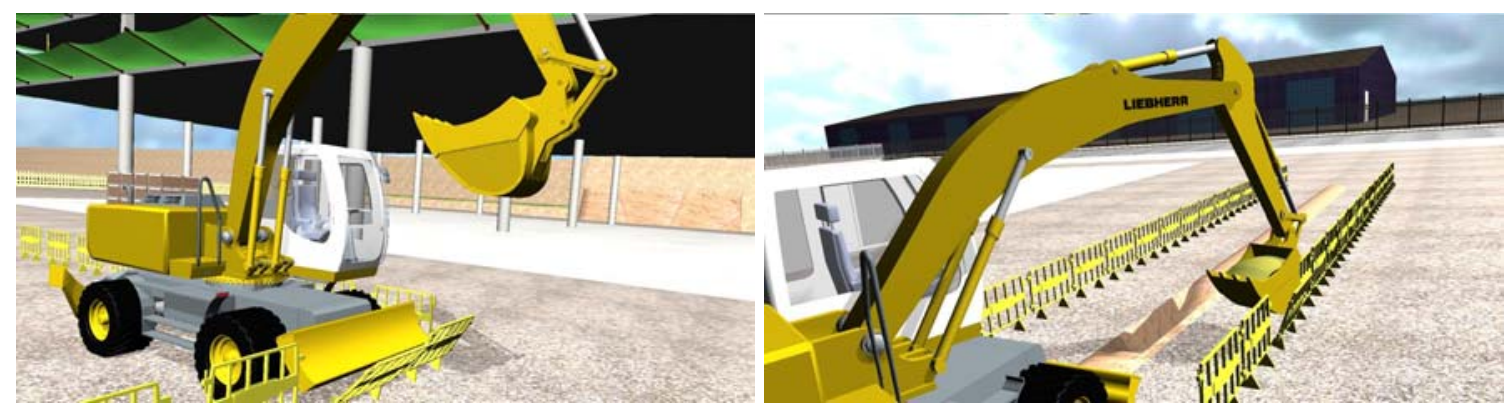

Figure 8: Interacting with movable objects: breaking down fences and digging.

although this limit is rarely reached.

The excavator model has 17 degrees of freedom (DOF), shown in Table 1, 7 DOF are controlled by the operator, while the remaining $10 \mathrm{DOF}$ are free. At this moment, the actuated DOF are kinematically guided because the dynamics of the hydraulic actuator circuit has not been modeled yet; therefore, the operator controls the position of actuated DOF without any delay or inertial effects. However, a simplified dynamics has been implemented and the values of the Lagrange multipliers are verified to limit the force available in the hydraulic actuators according to the torques and lift capacities given by the manufacturer. Also the velocities and accelerations of these kinematically guided DOFs have been adjusted to match the technical specifications of the real machine. The motion of the non-actuated DOF is determined by the forces applied to the model:

1. Weight of the machine parts and the bucket load.

2. Tire contact forces, which consist of linear spring and damper elements for the normal forces, and the magic formula tire model for the tangential forces Ref. [18].

3. Tire torques applied with the accelerator and brake pedals.

4. Contact forces originated from the collision of the excavator with the terrain or the surrounding objects; the contact model was described in section 3 .

The excavator is placed in a working environment (in Fig.(6) standing on its legs and blade), where the operator can perform different training exercises: maneuvering, digging, material 

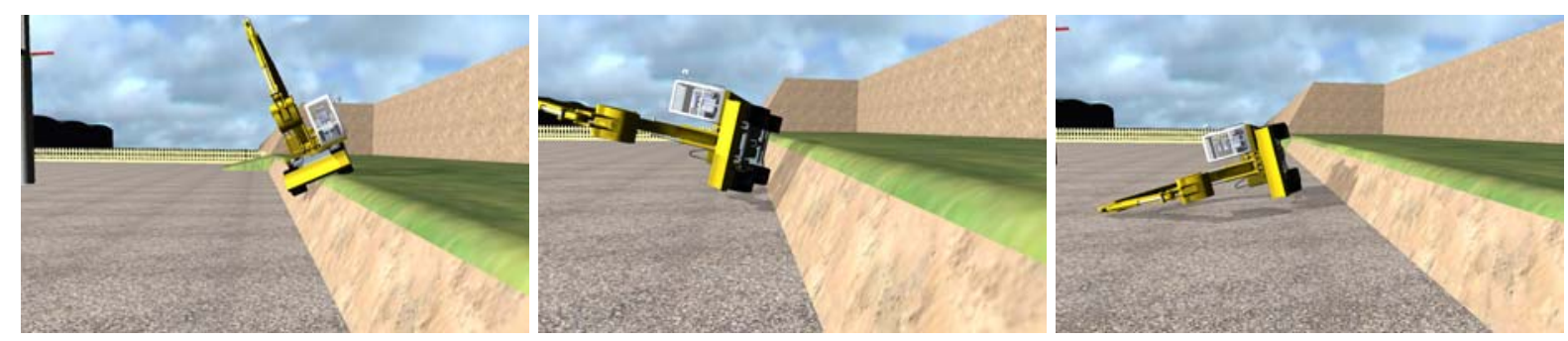

Figure 9: Excavator rollover.

\begin{tabular}{|l|c|}
\hline Motion & No. \\
\hline Actuated degrees of freedom & \\
\hline Boom, stick and bucket hydraulic cylinders & 3 \\
\hline Uppercarriage rotation & 1 \\
\hline Steering & 1 \\
\hline Stabilizer blade & 1 \\
\hline Outriggers & 1 \\
\hline TOTAL & 7 \\
\hline Non-actuated degrees of freedom & \\
\hline Undercarriage free motion & 6 \\
\hline Wheel rotation & 4 \\
\hline TOTAL & 10
\end{tabular}

Table 1: Degrees of freedom in the excavator model.

handling, etc. The excavator interacts with the environment in two ways (see Fig.( (7)): a) collisions with the scene objects and the terrain, which generate contact forces; and b) terrain excavation and loading with the bucket.

Some scene objects are fixed (e.g. buildings, terrain) while others are movable (e.g. fences) as can be seen in Fig. (8). In order to compute the dynamics of movable objects, they are introduced in/removed from the simulation only when the excavator approaches to/moves away from them; this technique makes possible to simulate in real-time working environments with a large number of movable objects.

The selected contact model delivers very realistic behavior and is able to simulate common events in the daily work of real excavators: slipping on slope terrains, stabilizing the machine with the blade and the outriggers, Fig. (6), using the arm for support or impulsion, Fig.( 7), moving objects with the bucket or blade, Fig.( 8 ), etc. or even other dangerous events not so common in the daily work, like rolling the excavator over Fig.( 9), etc.

\section{CONCLUSIONS}

- An integral solution to address contacts between solids in human-in-the-loop applications was described.

- The algorithm presented to integrate the equations of motion uses constant time-step and implicit integration, avoiding difficult and cumbersome detections of the precise instant of contact, modifications of the time step and re-starts of integration process. 
- The previous strategy of constant time step is very demanding for the integration algorithm since the algorithm has to overcome all the impacts and specially difficult situations present in the simulation without modifying the time step. The strategy has many advantages because of its simplicity but the main drawback is that the precission of the solution is not constant along the simulation.

- The proposed contact algorithm was tested in an academic example, showing that it observes the physical laws of the phenomenon and the performance of the algorithm is acceptable.

- The complete solution to address contacts was implemented in a real-time multibody model of a hydraulic excavator which is part of an excavator simulator, showing a wonderful behavior in the most common operation events of the daily work.

- The contact detection technique and computational aspects were taken into account along this work, which demonstrated to be crucial to achieve real-time performance.

\section{REFERENCES}

[1] H.M. Lankarani and P.E. Nikravesh. A contact force model with hysteresis damping for impact analysis of multibody systems. Journal of Mechanical Design, 112, 369-376, 1990.

[2] P. Flores, J. Ambrosio, J.C.P. Claro and H.M. Lankarani. Influence of the contact-impact force model on the dynamic response of multi-body systems. Proc. Inst. Mech. Eng. Part K: Journal of Multi-body Dynamic, 220, 21-34, 2006.

[3] P. Flores, J. Ambrosio, J.C.P. Claro and H.M. Lankarani. Kinematics and Dynamics of Multibody Systems with Imperfect Joints. Springer-Verlag, Berlin, Heidelberg, 2008.

[4] K.H. Hunt and F.R.E. Crossley. Coefficient of restitution interpreted as damping in vibroimpact. Journal of Applied Mechanics, 7, 440-445, 1975.

[5] F. Pfeiffer and C. Glocker. Multi-body dynamics with unilateral constraints. John Wiley and Sons, New York, 1996.

[6] K.A. Ismail and W.J. Stronge. Impact of Viscoplastic bodies: dissipation and restitution. Journal of Applied Mechanics, 75, 1-5, 2008.

[7] E.A. Butcher and D.J. Segalman. Characterizing damping and restitution in compliant impacts via modified K-V and higher-order linear viscoelastic models. Journal of Applied Mechanics, 67, 831-834, 2000.

[8] J. Garcia de Jalon and E. Bayo. Kinematic and dynamic simulation of multibody systems: The real-time challenge. Springer-Verlag, 1994.

[9] E. Bayo, J. García de Jalon, and M.A. Serna. A Modified Lagrangian Formulation for the Dynamic Analysis of Constrained Mechanical Systems. Computer Methods in Applied Mechanics and Engineering, 71, 183-195, 1988. 
[10] E. Bayo and R. Ledesma. Augmented Lagrangian and mass-orthogonal projection methods for constrained multibody dynamics. Nonlinear Dynamics, 9, 113-130, 1996.

[11] E. Eich-Soellner and C. Führer. Numerical methods in multibody dynamics. B.G. Teubner, Stuttgart, 1998.

[12] J. Cuadrado, R. Gutierrez, M.A. Naya and P. Morer. A comparison in terms of accuracy and efficiency between a MBS dynamic formulation with stress analysis and a non-linear FEA code. Int. Journal for Numerical Methods in Engineering, 51, 1033-1052, 2001.

[13] H.M. Lankarani and P.E. Nikravesh. Continuous Contact Force Models for Impact Analysis in Multibody Systems. Nonlinear Dynamics, 5, 193-207, 1994.

[14] J. A. Zukas, T. Nicholas, L. B. Greszczuk and D. R. Curran. Impact dynamics. John Wiley and Sons, New York, 1982.

[15] W. Goldsmith. Impact, The theory and physical behaviour of colliding solids. Edward Arnold Ltd, London, England, 1960.

[16] Y. Gonthier, J. McPhee, C. Lange and J.C. Piedboeuf. A Regularized Contact Model with Asymmetric Damping and Dwell-Time Dependent Friction. Multibody System Dynamics, 11, 209-233, 2004.

[17] J. D. Foley, A. van Dam, S. K. Feiner and J. Hughes. Introduction to Computer Graphics. Addison-Wesley Professional, 1993.

[18] H. B. Pacejka, and E. Bakker. "The Magic Formula Tyre Model," in Tyre models for vehicle dynamics analysis. H.B. Pacejka (ed.), Taylor and Francis, 1993.

[19] Liebherr-International Deutschland GmbH. Technical description A 924 C Litronic. http://www.liebherr.com/lh/en/, 2008. 